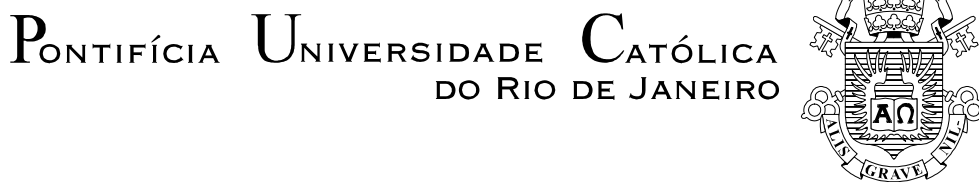

Felipe dos Santos Scofano

Desenvolvimento de um Elo Pneumático de 3 Graus de Liberdade para Manipuladores Robóticos Flexíveis

Dissertação de Mestrado

Dissertação apresentada como requisito parcial para obtenção do título de Mestre pelo Programa de PósGraduação em Engenharia Mecânica da PUC-Rio.

Orientador: Marco Antonio Meggiolaro 


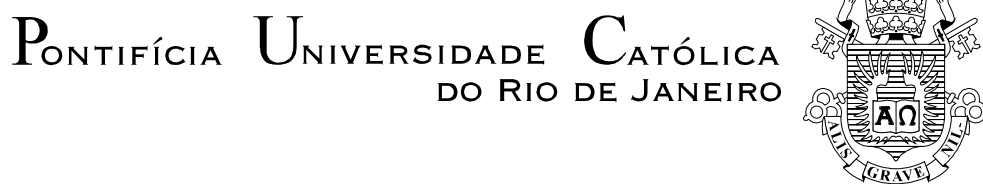

Felipe dos Santos Scofano

\title{
Desenvolvimento de um Elo Pneumático de \\ 3 Graus de Liberdade para Manipuladores \\ Robóticos Flexíveis
}

\begin{abstract}
Dissertação apresentada como requisito parcial para obtenção do título de Mestre pelo Programa de PósGraduação em Engenharia Mecânica da PUC-Rio. Aprovada pela Comissão Examinadora abaixo assinada.
\end{abstract}

Prof. Marco Antonio Meggiolaro

Orientador

Pontifícia Universidade Católica do Rio de Janeiro

Prof. Mauro Speranza Neto

Pontifícia Universidade Católica do Rio de Janeiro

D.Sc. Ricardo Rodrigues da Cunha Pinto

PETROBRAS

M.Sc. Alander Ornellas Machado PETROBRAS

Prof. José Eugênio Leal Coordenador Setorial do Centro

Técnico Científico - PUC-Rio

Rio de Janeiro, 18 de abril de 2006 
Todos os direitos reservados. É proibida a reprodução total ou parcial do trabalho sem autorização da universidade, do autor e do orientador.

Felipe dos Santos Scofano

Graduou-se em Engenharia de Controle e Automação, com ênfase em elétrica, na PUC-Rio (Pontifícia Universidade Católica do Rio de Janeiro). Desenvolve projetos vinculados a área de robótica.

Ficha Catalográfica

Scofano, Felipe dos Santos

Desenvolvimento de um elo pneumático de 3 graus de liberdade para manipuladores robóticos flexíveis / Felipe dos Santos Scofano; orientador: Marco Antonio Meggiolaro. - Rio de Janeiro : PUC, Departamento de Engenharia Mecânica, 2006.

200 f. : il. ; $30 \mathrm{~cm}$

Dissertação (mestrado) - Pontifícia Universidade Católica do Rio de Janeiro, Departamento de Engenharia Mecânica.

Inclui referências bibliográficas.

1. Engenharia mecânica - Teses. 2. Manipulador de longo alcance. 3. Elo flexível. 4. Músculo pneumático. I. Meggiolaro, Marco Antonio. II. Pontifícia Universidade Católica do Rio de Janeiro. Departamento de Engenharia Mecânica. III. Título. 


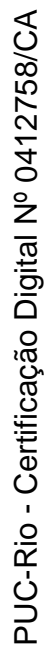

aos meus pais, Iara e Pedro Paulo 


\section{Agradecimentos}

Ao meu orientador Professor Marco Antonio Meggiolaro pela parceria e apoio para a realização deste trabalho.

Ao Professor Mauro Speranza pela confiança e reconhecimento.

Ao CNPq, à PUC-Rio e à PETROBRAS, pelos auxílios concedidos, sem os quais este trabalho não poderia ter sido realizado.

Ao meu amigo Felipe Belo por todo o apoio, companheirismo e cumplicidade.

Aos meus amigos Rodrigo Carvalho e Ilana Nigri por toda a colaboração.

Aos meus pais, pelo amor, pela educação, pelo apoio, atenção e carinho de todas as horas.

Aos meus colegas da PUC-Rio.

Aos professores que participaram da Comissão examinadora.

A todos os amigos e familiares que de uma forma ou de outra me estimularam ou me ajudaram. 


\section{Resumo}

Scofano, Felipe dos Santos. Desenvolvimento de um Elo Pneumático de 3

Graus de Liberdade para Manipuladores Robóticos Flexíveis. Rio de

Janeiro, 2006. 200p. Dissertação de Mestrado - Departamento de

Engenharia Mecânica, Pontifícia Universidade Católica do Rio de Janeiro.

Recentemente, grande interesse tem se voltado na robótica para o conceito de manipuladores flexíveis. Estes dispositivos apresentam uma coluna vertebral deformável continuamente, em oposição aos tradicionais manipuladores robóticos elo/junta/elo com elos rígidos. Sistemas flexíveis oferecem um aumento em potencial da capacidade de interação com o ambiente, estando aptos a se ajustarem às limitações do meio através de sua deformação. Robôs flexíveis oferecem possibilidades atrativas para o uso em diversas aplicações, como em posicionamento em ambientes complexos com obstáculos, endoscópios ativos, e manuseamento de materiais frágeis. $\mathrm{O}$ uso de polímeros, em particular elastômeros, tem sido explorado nestes manipuladores para promover simplicidade de operação e menor rigidez, necessária para uma interação homemmáquina com maior segurança. Usufruindo-se destes conceitos, esta dissertação aborda o desenvolvimento de um manipulador pneumático flexível de longo alcance. O manipulador é composto por uma estrutura modular, formada por vários elos ligados serialmente, permitindo que em sua extremidade sejam acopladas ferramentas que auxiliem a execução de diferentes tarefas. O sistema é baseado em um atuador pneumático denominado Músculo Artificial Pneumático (Pneumatic Artificial Muscles, PAM). Ao serem pressurizados, estes dispositivos se contraem, exercendo uma força em sua extremidade proporcional à pressão aplicada. A movimentação do manipulador desenvolvido é obtida a partir da diferença de pressão entre câmaras independentes localizadas em seu interior. Modelos analíticos dos sistemas desenvolvidos foram elaborados. O controle do manipulador é feito a partir de servoválvulas pneumáticas controladas por computador. Experimentos foram realizados para verificar os modelos desenvolvidos. O sistema desenvolvido pode ser aplicado à tarefa de inspeção interna de reservatórios de combustíveis. Inspeções internas atualmente requerem um completo esvaziamento do reservatório, se tornando muito trabalhosas e resultando em altos custos. Uma versão do manipulador é adaptada para executar esta tarefa sem a necessidade de esvaziar os tanques, devido à segurança intrínseca do sistema pneumático.

\section{Palavras-chave}

Manipulador de longo alcance, elo flexível, músculo pneumático artificial 


\section{Abstract}

Scofano, Felipe dos Santos. Development of a Three Degree-of-Freedom

Pneumatic Link for Flexible Robotic Manipulators. Rio de Janeiro, 2006. 200p. MSc. Dissertation - Departamento de Engenharia Mecânica, Pontifícia Universidade Católica do Rio de Janeiro.

Recently, the concept of flexible manipulators has attract great interest. These devices present a continuously deforming vertebral column, in opposition to the traditional robotic manipulators link/joint/link with rigid links. Flexible systems offer a potential increase in the capacity of interaction with the environment, being apt to adjust itself to the constrants through its deformation. Flexible robots offer attractive possibilities for usage in many applications, as complex environments positioning with active obstacles, endoscopies, and manipulating fragile materials. Polymers, specially elastomers, have been explored in these manipulators to guarantee simple operation and minor rigidity, necessary for a higher security man-machine interaction. Making a good use of these conceptions, this dissertation presents the development of a long-reach flexible pneumatic manipulator. The manipulator is composed of a modular structure, formed by links attached serially, allowing tools to be connected in its end-point for assistence in different tasks. The system is based on a pneumatic actuator called Pneumatic Artificial Muscle (PAM). When pressurized, these devices contract themselves, exerting a proportional force in its end-points proportional to the applied pressure. The manipulator's motion is obtained from the pressure difference between the independent chambers located in its interior. Developed systems analytical models have been elaborated. Pneumatic valves, commanded by computer, control the manipulator. Experiments have been carried through to test the developed models. The developed system can be applied to internal inspection of fuel tanks. Internal inspections currently require a complete tank ullage, becoming very laborious and resulting in high costs. A manipulator's version is adapted to execute this task in a full fueled tank, due to intrinsic security of the pneumatic system.

\section{Keywords}

Long-reach manipulator, flexible link, artificial pneumatic muscle 


\section{Sumário}

1 Introdução 17

1.1. Motivação 19

1.2. Objetivo 21

1.3. Organização da Tese 22

2 Sistemas Robóticos Flexíveis 23

2.1. Princípios de Atuação 23

2.2. Sistemas Existentes 26

2.3. Músculos Pneumáticos Artificiais 32

2.3.1. Propriedades 33

2.3.2. Tipos de Músculos $\quad 37$

$\begin{array}{ll}\text { 2.3.3. Aplicações } & 47\end{array}$

3 Concepção e Modelagem do Sistema 48

3.1. Mangueiras sem Reforço $\quad 51$

3.1.1. Modelagem 52

3.1.2. Experimento $\quad 57$

3.2. Mangueiras Reforçadas por Anéis $\quad 59$

3.2.1. Modelagem $\quad 59$

3.2.2. Experimento 65

3.3. Mangueiras Reforçadas por Fios 73

3.3.1. Modelagem 74

3.3.2. Experimento $\quad 75$

3.4. Músculo MAS-10 da FESTO 77

3.4.1. Experimentos 79

3.5. Montagem dos Elos 81

3.5.1. Modelo 3D 81

3.5.2. Desenvolvimento de Espaçadores 85

$\begin{array}{ll}\text { 3.5.3. Elo com Corrugado } & 107\end{array}$ 
4 Resultados Experimentais com o Manipulador 112

4.1. Montagem do sistema 112

$\begin{array}{ll}\text { 4.2. Modelagem } & 119\end{array}$

$\begin{array}{ll}\text { 4.3. Resultados Experimentais } & 123\end{array}$

5 Aplicação em Inspeção de Tanques de Combustível 126

5.1. Sistemas de Inspeção Existentes 126

5.2. Descrição do Sistema 127

5.3. Tipos de Tanques 129

5.3.1. Tanques de Teto Fixo (Fixed Roof) 130

5.3.2. Tanques de Teto Móvel (Lifting Roof) 132

5.3.3. Tanques de Teto Fixo com Diafragma Flexível (Diaphragm) 132

5.3.4. Tanques de Teto Flutuante (Floating-Roof) 133

5.3.5. Tanques de Postos de Serviço 138

5.3.6. Bocais e Acessórios 139

$\begin{array}{ll}\text { 5.4. Aplicação } & 145\end{array}$

6 Conclusões e Trabalhos Futuros 146

6.1. Contribuições neste Trabalho 146

$\begin{array}{ll}\text { 6.2. Sugestões para Trabalhos Futuros } & 149\end{array}$

$\begin{array}{ll}\text { Referências bibliográficas } & 150\end{array}$

$\begin{array}{lr}\text { ANEXO } & 153\end{array}$ 


\section{Lista de figuras}

Figura 1: Esquema ilustrando a construção dos dedos de borracha 24

Figura 2: Esquema do prendedor macio $\quad 25$

Figura 3: Esquema do rubbertuator fabricado pela Bridgestone 25

Figura 4: Músculo pneumático MAS da FESTO, disponível em três diâmetros, 10, 20 e $40 \mathrm{~mm} \quad 26$

Figura 5: Robô contínuo tipo tentáculo $\quad 27$

Figura 6: Garra hidráulica do manipulador AMADEUS 28

Figura 7: Fole cilíndrico de metal corrugado; pressão interna

$\begin{array}{ll}\text { causando expansão longitudinal } & 29\end{array}$

Figura 8: Tentáculo KSI 29

Figura 9: Dedos de borracha atuados pneumaticamente segurando e apertando objetos $\quad 30$

Figura 10: Robô AirBug com atuadores MAS-20 da FESTO 31

Figura 11: Braço pneumático com atuadores MAS-10 da FESTO 31

Figura 12: Braço protético com músculos de McKibben 32

Figura 13: Diagrama isobárico de força-contração do

Músculo Artificial Pneumático 34

Figura 14: Tubo reforçado com malha de fibras trançadas 39

Figura 15: Músculo Trançado (Músculo de McKibben) 39

Figura 16: Músculo com Pregas; totalmente esticado e inflado 40

Figura 17: Músculo de Yarlott 41

Figura 18: ROMAC, versão padrão (a) e versão em miniatura (b) 42

Figura 19: Músculo de Kukolj 43

Figura 20: Diferentes protejos do Músculo de Morin de acordo com a pressão utilizada; (a) protejo para sobrepressão (vista do corte transversal longitudinal), (b) protejo para subpressão e (c) com membranas concêntricas $\quad 44$

Figura 21: Músculo do tipo Baldwin $\quad 45$

Figura 22: Músculo Paynter Hiperbólico 46

Figura 23: Torção de Kleinwachter $\quad 47$ 
Figura 24: Representação de um elo do manipulador composto

por duas câmaras

Figura 25: Modelo 2D do manipulador pneumático com 3 elos

em série, sendo a posição da sua extremidade representada pelas

cordenadas $x_{e}, y_{e}$ e $\alpha_{e}$

Figura 26: Elo do manipulador aproximado por um arco de círculo 49

Figura 27: Mangueiras flexíveis diversas 52

Figura 28: Mangueira sem pressão 52

Figura 29: Mangueira pressurizada 53

Figura 30: Perfil da mangueira com parede fina 53

Figura 31: Forças resultantes geradas pela tensão axial 53

Figura 32: Forças resultantes geradas pela tensão circunferencial 54

Figura 33: Perfil da mangueira com parede grossa 56

Figura 34: Ruptura da mangueira de silicone pelo excesso de pressão aplicada 58

Figura 35: Mangueira de silicone pressurizada reforçada com anéis circulares 59

Figura 36: Tensões radiais presentes na mangueira 60

Figura 37: Anéis reforçando a mangueira, espaçados de uma distância $\Delta x \quad 63$

Figura 38: Tensões agindo sobre o anel 63

Figura 39: Anéis flexíveis $\quad 66$

Figura 40: Mangueira de silicone com os anéis flexíveis 66

Figura 41: Comprimento da mangueira de silicone com anéis

$\begin{array}{ll}\text { flexíveis em função da pressão interna inserida } & 67\end{array}$

Figura 42: Escorregamento dos anéis durante a pressurização da

mangueira

Figura 43: Anéis cortados a partir de uma mangueira rígida

reforçada com fibras

Figura 44: Desenho em corte longitudinal da área deformável

(em azul) da mangueira em função do tipo de anel, circular ou quadrado $\quad 70$

Figura 45: Anéis rígidos $\quad 70$

Figura 46: Mangueira de silicone montada com anéis rígidos 71

Figura 47: Anéis rígidos amontoados durante a curvatura da mangueira 72

Figura 48: Mangueira de silicone montada com anéis rígidos

e flexíveis intercalados 
Figura 49: Anéis rígidos e flexíveis durante a curvatura da mangueira

Figura 50: Ilustração da deformação de uma mangueira envolta por um fio 74

Figura 51: Mangueira de silicone envolta pelo fio rígido de polipropileno 75

Figura 52: Deformação axial da mangueira reforçada com o fio de

polipropileno

Figura 53: Músculo pneumático MAS-20 da FESTO, totalmente

contraído na parte superior da figura e no seu comprimento inicial na parte inferior

Figura 54: Diagrama da faixa de operação do MAS -10 78

Figura 55: Montagem do experimento com o músculo 79

Figura 56: Gráfico da faixa de operação do MAS-10 a partir dos dados experimentais

Figura 57: Gráfico comparativo entre as curvas fornecidas pelo

fabricante e os dados experimentais

Figura 58: Esquema de um elo do manipulador com as suas

três câmaras independentes

Figura 59: Modelo analítico de cada elo

Figura 60: $1^{\text {a }}$ Geração, mangueiras siliconadas presas com cola de poliuretano reforçadas com abraçadeiras $\quad 86$

Figura 61: $1^{\text {a }}$ Geração, outra vista 86

Figura 62: Extremidades das mangueiras, fechadas com espigões e $\begin{array}{ll}\text { presas através de abraçadeiras } & 87\end{array}$

Figura 63: Desenho de um corte em perfil da $1^{\mathrm{a}}$ geração 87

Figura 64: $2^{\mathrm{a}}$ Geração, mangueiras de silicone revestidas com cola

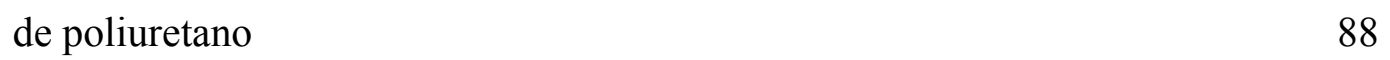

Figura 65: Desenho de um corte em perfil da 2a geração 88

Figura 66: $3^{\text {a }}$ Geração, mangueiras de silicone reforçadas

com fio de polipropileno, revestidas com cola de poliuretano

Figura 67: Rompimento da cola de poliuretano devido à força $\begin{array}{ll}\text { exercida pelos músculos } & 90\end{array}$

Figura 68: Desenho de um corte em perfil da $3^{\text {a }}$ geração $\quad 90$

Figura 69: $4^{\mathrm{a}}$ Geração, mangueiras de silicone reforçadas com anéis flexíveis de butadieno-acrilonitrila, agrupadas com abraçadeiras 
Figura 70: Desenho de um corte em perfil da $4^{\mathrm{a}}$ geração 92

Figura 71: Espaçadores de Fenolite 92

Figura 72: $4^{\mathrm{a}}$ Geração, mangueiras de silicone reforçadas com

anéis flexíveis e rígidos, agrupadas com espaçadores de Fenolite

Figura 73: Desenho de um corte em perfil da $4^{\mathrm{a}}$ geração com

anéis rígidos (representados pelos círculos amarelo, verde e

azul) e com o espaçador de Fenolite

Figura 74: 5a Geração, músculos MAS -10 da FESTO revestidos

com fita flexível 95

Figura 75: Conexões dos MAS -10 95

Figura 76: Desenho de um corte em perfil da $5^{\mathrm{a}}$ geração 96

Figura 77: Amostra do espaçador feito de mangueiras siliconadas 96

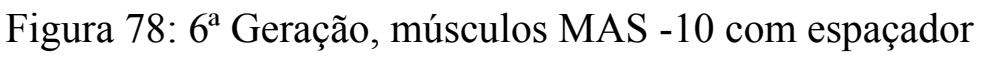

de mangueira siliconada, revestidos com fita flexível 97

Figura 79: $6^{\text {a }}$ Geração, músculos MAS -10 com espaçador

de mangueira siliconada, revestidos com fita flexível 98

Figura 80: Desenho de um corte em perfil da 6 $6^{\text {geração }} 98$

Figura 81: Flambagem de um dos músculos devido à pressão interna

$\begin{array}{ll}\text { muito baixa } & 98\end{array}$

Figura 82: Comparação entre a curvatura do elo e um círculo de raio $R \quad 99$

Figura 83: Espaçador rígido de polipropileno (branco) e espaçador

$\begin{array}{ll}\text { flexível de silicone (preto) } & 100\end{array}$

Figura 84: Desenho do espaçador com as suas funções 100

Figura 85: $7^{\mathrm{a}}$ Geração, músculos MAS-10 com espaçadores de

polipropileno e de silicone intercalados

Figura 86: Movimentação desordenada dos músculos no interior

dos espaçadores gerando formas aleatórias. 102

Figura 87: Desenho de um corte em perfil da $7^{\mathrm{a}}$ geração 102

Figura 88: Demonstração da baixa rigidez axial do elo 103

Figura 89: Dimensões do termo retrátil 104

Figura 90: Montagem do termo retrátil no elo 104

Figura 91: Comparação entre a curvatura do elo e um círculo

representando a curvatura de gerações anteriores sob mesma 
diferença de pressão

Figura 92: Montagem da atadura elástica no elo, com

espaçadores internos siliconados

Figura 93: Elo revestido com atadura elástica com acabamento

de tubo termo-retrátil

106

Figura 94: Comparação entre a curvatura de protótipos iguais com

revestimentos diferentes (do menor ao maior raio: elo sem

revestimento, revestido com atadura elástica e revestido com tubo

termo-retrátil)

107

Figura 95: Mangueira de poliuretano revestida com fio de aço cobreado 108

Figura 96: Tubo corrugado de PTFE 109

Figura 97: Tubo corrugado de PTFE com suas dimensões características 109

Figura 98: Mangueira de PVC modelo KV 110

Figura 99: Elo reforçado com mangueira de poliuretano externa 111

Figura 100: Detalhe da mangueira de poliuretano externa 111

Figura 101: Protótipo final utilizado nos experimentos com as

servo-válvulas

Figura 102: Protótipo final; detalhe da montagem com a mangueira

$\begin{array}{ll}\text { de poliuretano reforçada } & 113\end{array}$

Figura 103: Válvula proporcional controlada por tensão (Bosch-Rexroth) 115

Figura 104: Compressor CSI 7,4/50 (SCHULZ) 115

Figura 105: Placa ISA Bus Servo I/O Card utilizada nos experimentos 116

Figura 106: Interface do programa de controle desenvolvido em LabView 117

$\begin{array}{ll}\text { Figura 107: Bancada de experimento } & 118\end{array}$

Figura 108: Equipamentos utilizados nos experimentos (da esquerda

para a direita - servoválvulas, fonte de alimentação e o sistema

$\begin{array}{ll}\text { manual de válvulas) } & 118\end{array}$

Figura 109: Aproximação da curvatura do elo por um arco de círculo 119

Figura 110: Corte transversal do elo, com os músculos pneumáticos

em preto e o espaçador em azul

Figura 111: Obtenção de medidas da extremidade do manipulador

Figura 112: Esquema do manipulador em operação no interior de

um reservatório de combustível 
Figura 113: Tanques de armazenamento

Figura 114: Teto cônico suportado. Componentes da estrutura

de sustentação. Vigas radiais, vigas transversais e colunas 130

Figura 115: Teto fixo cônico 131

Figura 116: Teto fixo curvo 131

Figura 117: Teto fixo em gomos 132

Figura 118: Teto móvel 133

Figura 119: Teto flutuante simples 134

Figura 120: Teto flutuante com flutuador 135

Figura 121: Teto flutuante Buoyroof 135

Figura 122: Teto flutuante duplo 136

$\begin{array}{ll}\text { Figura } 123 \text { Tanques de posto de serviço } & 138\end{array}$

Figura 124: Tanque e acessórios -Terminologia 141

Figura 125: Escotilhas de medição 143

Figura 126: Desenho esquemático de um tanque jaquetado, pleno,

com seus bocais e acessórios 144 


\section{Lista de tabelas}

Tabela 1: Comprimento da mangueira de silicone em função da

pressão interna inserida

Tabela 2: Comprimento da mangueira de silicone em função da pressão interna inserida

Tabela 3: Dados experimentais do MAS-10

Tabela 4: Comprimento do elo em função da pressão interna inserida

Tabela 5: Especificações técnicas do termo retrátil

104

Tabela 6: Especificações da mangueira de poliuretano 108

Tabela 7: Especificações do tubo de PTFE 109

Tabela 8: Especificações da mangueira de PVC Kanaflex KV 110

Tabela 9: Principais características da válvula proporcional 114

Tabela 10: Principais características do compressor $\quad 115$

Tabela 11: Posicionamento $x y$ da extremidade do manipulador 125

Tabela 12: Tipo de tanque em função do produto armazenado 137

Tabela 13: Capacidade e dimensões de tanque 139

Tabela 14: Bocas de visita, portas de limpeza e drenos de fundo dos tanques 142 